\title{
Expression of hepatitis $C$ virus nonstructural 4B in transgenic mice
}

\author{
Ai-Guo Wang ${ }^{1}$, Hyung-Bae Moon ${ }^{2}$, \\ Jin-Man $\mathrm{Kim}^{3}$, Soon-Bong Hwang ${ }^{4}$, \\ Dae-Yeul $\mathrm{Yu}^{1,5}$ and Dong-Seok Lee ${ }^{1,5}$ \\ ${ }^{1}$ Laboratory of Human Genomics \\ Korea Research Institute of Bioscience \\ and Biotechnology (KRIBB) \\ Daejeon 305-806, Korea \\ ${ }^{2}$ Department of Pathology and Institute of Medical Science \\ Wonkwang University School of Medicine \\ Iksan, Chonbuk 570-749, Korea \\ ${ }^{3}$ Department of Pathology, College of Medicine \\ Chungnam National University \\ Daejeon 301-131, Korea \\ ${ }^{4}$ Ilsong Institute of Life Science \\ Hallym University \\ Chuncheon 200-702, Korea \\ ${ }^{5}$ Corresponding author: Tel, 82-42-860-4349; \\ Fax, 82-42-860-4608; E-mail, lee10@kribb.re.kr
}

Accepted 12 May 2006

Abbreviations: HCV, hepatitis C virus; NS4B, nonstructural 4B

\begin{abstract}
Hepatitis $\mathrm{C}$ virus (HCV) is a pathogen that is of great medical significance in chronic hepatitis, liver cirrhosis, and hepatocellular carcinoma worldwide. Although the HCV proteins have been intensively investigated over the past decade, the biochemical functions of the NS4B protein are still largely unknown. To investigate NS4B as a potential causative agent of liver disease, transgenic mice expressing the NS4B protein in liver tissue were produced. The transgenic animals were phenotypically similar to their normal littermates for up to 18 months of age. Our results suggest that the HCV NS4B protein is not directly cytopathic or oncogenic in our transgenic mice model.
\end{abstract}

Keywords: hepacivirus; liver; mice, transgenic; NS4 protein, hepatitis $\mathrm{C}$ virus; viral nonstructural proteins

\section{Introduction}

The hepatitis $\mathrm{C}$ virus $(\mathrm{HCV})$ is a major cause of chronic hepatitis, liver cirrhosis, and hepatocellular carcinoma worldwide (1997, 1999, Moriwaki, 2002), and chronic hepatitis $C$ has become a serious global medical problem and a considerable burden on health care systems (Tan et al., 2001). Unfortunately, neither a vaccine nor any other means of effective chemotherapy is available to control $\mathrm{HCV}$ infection at this time. Furthermore, research efforts have been hampered by the lack of both a cell culture system that allows for efficient HCV replication and small animal models that either support replication of the $\mathrm{HCV}$ or mimic the pathologic features of chronic hepatitis C in humans (Lemon et al., 1998). Transgenic animal models have contributed pivotal information on hepatitis virus-mediated pathogenesis (Hanahan, 1988; Chisari, 1995; Milich, 2000). Studies involving HCV Core transgenic mice have suggested that core protein may play an important role in $\mathrm{HCV}$ induced steatosis and HCC (Moriya et al., 1997; Moriya et al., 1998). Recently, Lerat et al. reported that transgenic mice expressing complete HCV viral polyprotein developed steatosis and liver cancer as early as 13 months of age (Lerat et al., 2002). The findings suggested that the expression of structural proteins enhances a low background of steatosis in C57BL/6 mice, while the additional low-level expression of nonstructural proteins increases the risk of cancer.

The functions of the NS4B protein, which is one of the nonstructural proteins of the $\mathrm{HCV}$, are largely unknown. The locations of the NS4B protein have been identified. It is mainly associated with the endoplasmic reticulum (ER) and is partly contained in the cytoplasm (Lundin et al., 2003). However, the translocation of NS4B to the nucleus has also been suggested (Westaway et al., 1997). One function of NS4B related to $\mathrm{HCV}$ replication was reported (Piccininni et al., 2002; Hanley et al., 2003). In addition, the inhibitory function of NS4B on host protein synthesis during translation (Florese et al., 2002, Kato et al., 2002) may contribute to the support of HCV survival in host cells. In addition, NS4B inhibited p21 $1^{\text {Waf } 1 / C i p 1}$ and p53 (Florese et al., 2002), suggesting its potential function in HCVinduced pathological changes in the liver. The mutation of NS4B has also been found to significantly reduce pathological injury induced by bovine viral diarrhea virus (BVDV) (closely related to $\mathrm{HCV}$ ) in cells (Qu et al., 2001). Moreover, NS4B in association with Ha-ras plays an important role in the 
malignant transformation of cells (Park et al., 2000). However, in vivo experiments have not been performed to contribute to these in vitro studies.

In the present study, we report the generation of transgenic mice, the expression of HCV NS4B, and the histological findings of the liver. Our results indicate that HCV NS4B is not cytopathic to cells in vivo.

\section{Materials and Methods}

\section{Generation and identification of transgenic mice}

To generate the $\mathrm{pHEX/NS4B}$ vector, a 0.78-kb fragment containing HCV genotype 1b NS4B (Korea strains) was subcloned into the $\mathrm{Nco}$ I/Bgl II sites of $\mathrm{pHEX}$ to replace the $\mathrm{X}$-gene (Figure $1 \mathrm{~A}$ ). The plasmid $\mathrm{pHEX}$ is a previously reported vector prepared by us that expresses the HBX gene under authentic promoter control (Yu et al., 1999). Injectable DNA containing $H B V$ enhancer, $N S 4 B$, and SV40 poly $A$ sequences was obtained by the removal of phagemid sequences from pUC118 at Kpn I/Hind III sites. DNA was microinjected into fertilized mouse eggs of hybrid BCF1 (C57BL/6J 우 x $\mathrm{CBA} / \mathrm{J}$ ), and the injected eggs were transferred into pseudopregnant recipients as previously described
(Yu et al., 1999). Potential founders were analyzed by polymerase chain reaction (PCR) and Southern blot analysis of tail DNA. Transgenic lineages were transmitted to the $\mathrm{C} 57 \mathrm{BL} / 6 \mathrm{~J}$ inbred line by mating with C57BL/6J mice. The mice were housed in a controlled, specific pathogen-free environment and were maintained in accordance with the guidelines of the Institutional Animal Care and Use Committee, Korea Research Institute of Bioscience and Biotechnology (KRIBB, Daejeon, South Korea).

\section{RT-PCR analysis}

We performed RT-PCR to detect the expression of the transgene in transgenic mouse tissues. The mice were killed, and the tissue samples were immediately frozen in liquid nitrogen. Total RNA was isolated from mouse tissues using the TRIzol reagent (Invitrogen Life Technologies Inc., Carlsbad, CA). RT-PCR was performed using a reverse transcription system (Promega Corp., Madison, WI) according to the manufacturer's instructions. The primers used to detect the expression of the transgene were the sense primer, 5'-GCCTCACACCTTCCTTAC-3', and the anti-sense primer, 5'-CAGGATGTCCACAAGCAC-3'. A separate RT-PCR primer was used to detect GAPDH as a loading control.
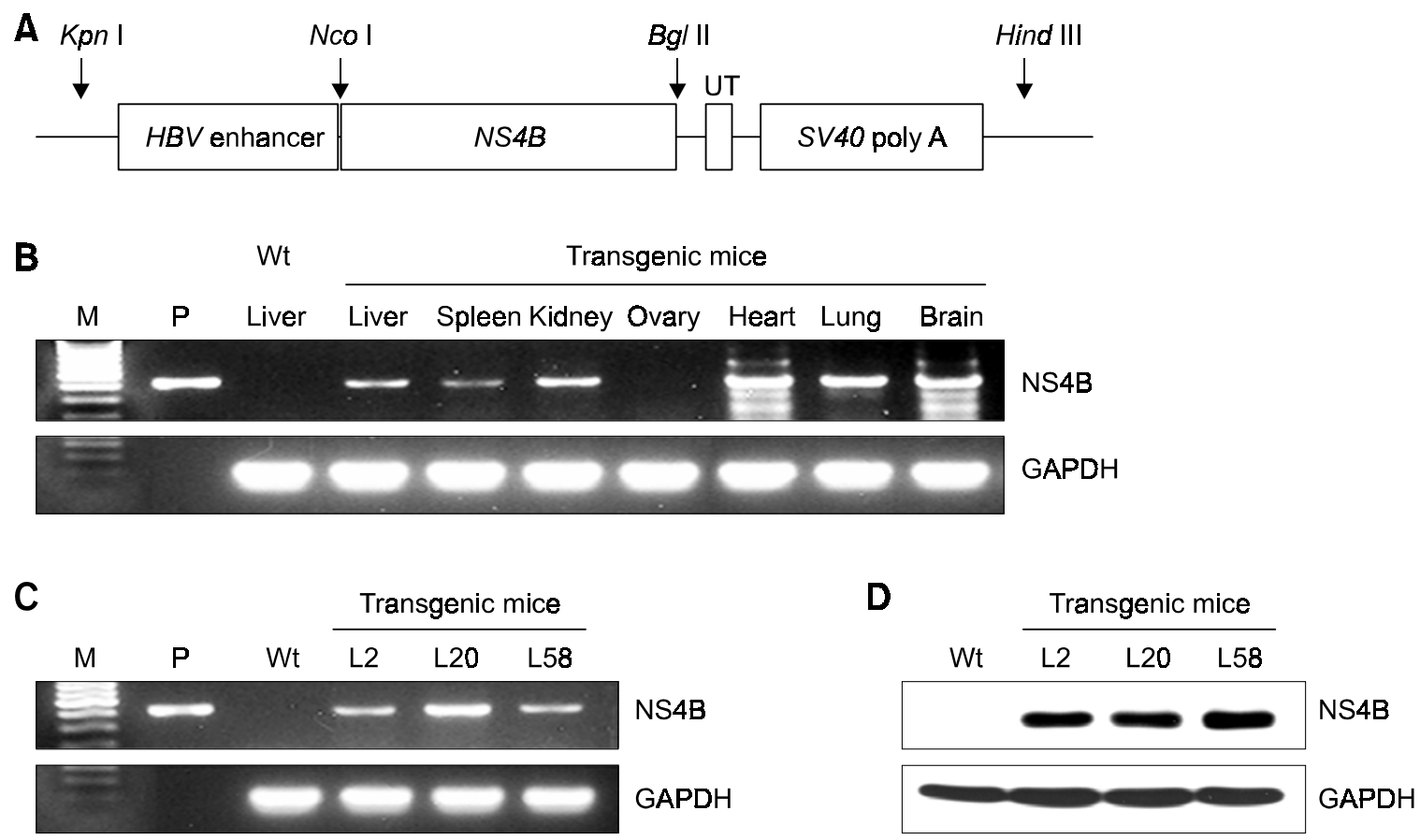

Figure 1. Vector construction and transgene expression. (A) Schematic of the expression vector $p H E X / N S 4 B$. (B) The expression of NS4B in the tissues of lineage 2 was analyzed by RT-PCR. (C) The expression of NS4B in liver tissues of transgenic lineages was analyzed by RT-PCR. (D) The expression of HCV NS4B protein level was measured performed in liver tissues of transgenic lineages by Western blot analysis. GAPDH was used as a loading control. M, size marker; P, positive control; Wt, normal littermate; L2, lineage 2; L20, lineage 20; L58, lineage 58. 


\section{Western blot analysis}

Tissue lysates were prepared by homogenizing tissues that had been freshly retrieved or flash-frozen in lysis buffer. For Western blot analysis, equal amount $(20 \mu \mathrm{g})$ of proteins were resolved by SDSPAGE and transferred to a polyvinylidene difluoride membrane (Millipore, Billerica, MA). The membranes were probed with the primary and secondary antibodies: mouse monoclonal anti-NS4B antibody (Advanced Immunochemical Inc.); anti-GAPDH (Trevigen, Gaithersburg, MD); horseradish peroxidase (HRP)-conjugated goat anti-rabbit IgG antibodies (Santa Cruz Biotechnology, Santa Cruz, CA); and HRP-conjugated goat anti-mouse IgG (Amersham Biosciences, Buckinghamshire, UK). Positive bands were detected using an enhanced chemiluminescence system (Pierce, Rockford, IL).

\section{Histopathological diagnoses and immunohistochemical staining}

Tissue samples were fixed in 10\% neutral buffered formalin, embedded in paraffin, sectioned, and stained with hematoxylin and eosin (H\&E) according to standard methods. The histopathological diagnoses were based on the criteria described by Frith and Ward (Frith et al., 1979). The immunohistochemical studies were performed on formalin-fixed, paraffinembedded, $4 \mu \mathrm{m}$-thick tissue sections using the streptavidin-biotin peroxidase complex method, as previously described (Yu et al., 1999). The primary antibody used was the mouse monoclonal antiNS4B antibody (Advanced Immunochemical Inc.).

\section{Results}

\section{Generation of NS4B transgenic mice}

To generate the transgenic mice, the HCV NS4B genomic region under the control of the HBV enhancer was microinjected into fertilized oocytes from BCF1 hybrid mice. Four founders containing the HBV enhancer-HCV NS4B sequence were identified. Among them, three were selected for the establishment of the transgenic lineage by backcrossing against the C57BL/6J parental strain.

\section{Expression of the NS4B transgene}

In order to determine the expression of the HCV NS4B transgene at the mRNA level in transgenic mouse tissues, RT-PCR was performed. The results showed that NS4B was expressed widely in the tissues of transgenic mice, including the liver, but was not expressed in normal control littermates (Figure 1B and $\mathrm{C}$ ). This expression pattern was similar to the pattern that appeared in the transgenic mice expressing the HBV $X$ protein and HCV core protein under the direction of the HBV enhancer sequence in our previous study (Yu et al., 1999; Wang et al., 2004). To find out if the mRNA was translated to the protein in the liver, Western blot analysis and immunohistostaining were performed. The Western blot data showed that HCV NS4B protein was substantially expressed in liver tissues (Figure 1D). In addition, the immunohistostaining showed that the expression of HCV NS4B protein was present throughout the cytoplasmic of hepatocytes in transgenic mice (Figure 2).
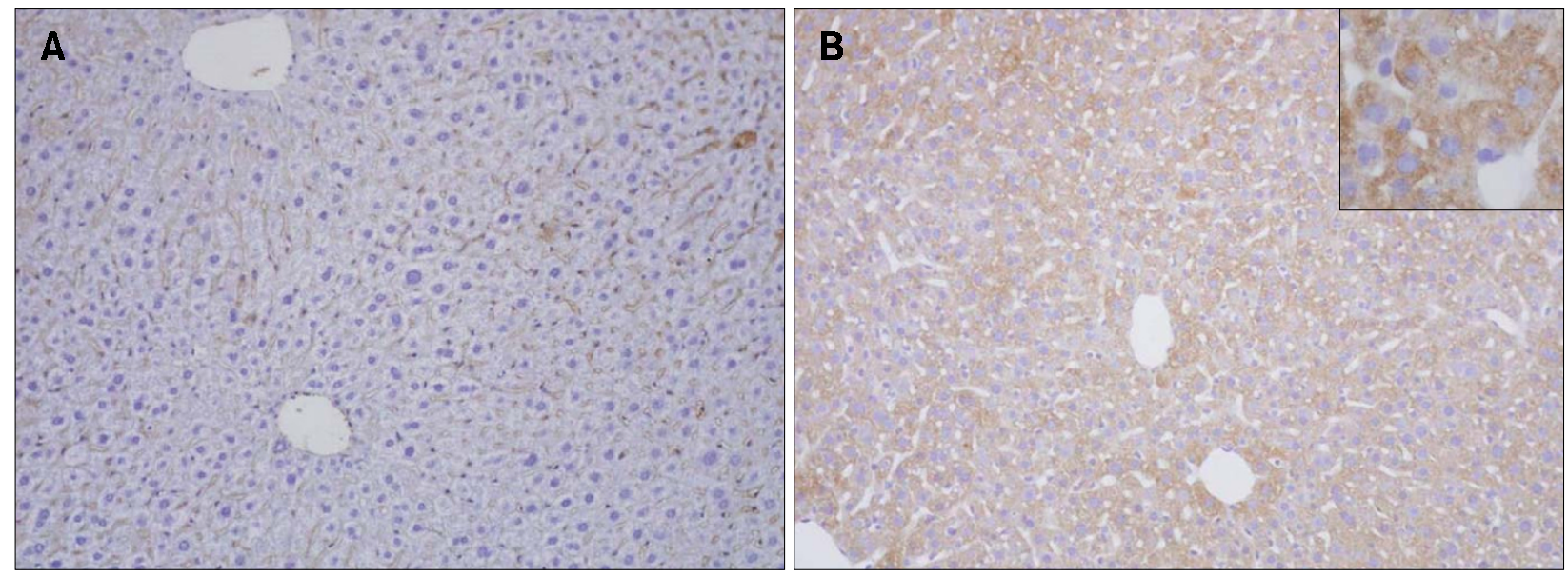

Figure 2. Immunohistochemical analysis of HCV NS4B in the livers of normal littermates (A) and transgenic mice (B) using a specific monoclonal antibody. NS4B antibody staining is predominantly in the cytoplasm of transgenic hepatocytes showed in $B$ at magnification $20 \times$ and $200 \times$ (upper-right corner). Magnification for $(A)$ is $20 \times$. 
Table 1. Histological summary of HCV NS4B transgenic mouse lineages.

\begin{tabular}{ccccc}
\hline Transgenic line & $\begin{array}{c}\text { Number of mice } \\
\text { examined }\end{array}$ & Age (months) & Liver abnormalities & $\begin{array}{c}\text { Other organs } \\
\text { abnormalities }\end{array}$ \\
\hline Line 2 & 14 & $9-18$ & None & None \\
Line 20 & 10 & $9-18$ & None & None \\
Line 58 & 11 & $9-18$ & None & None \\
Normal & 15 & $9-18$ & None & None \\
\hline
\end{tabular}
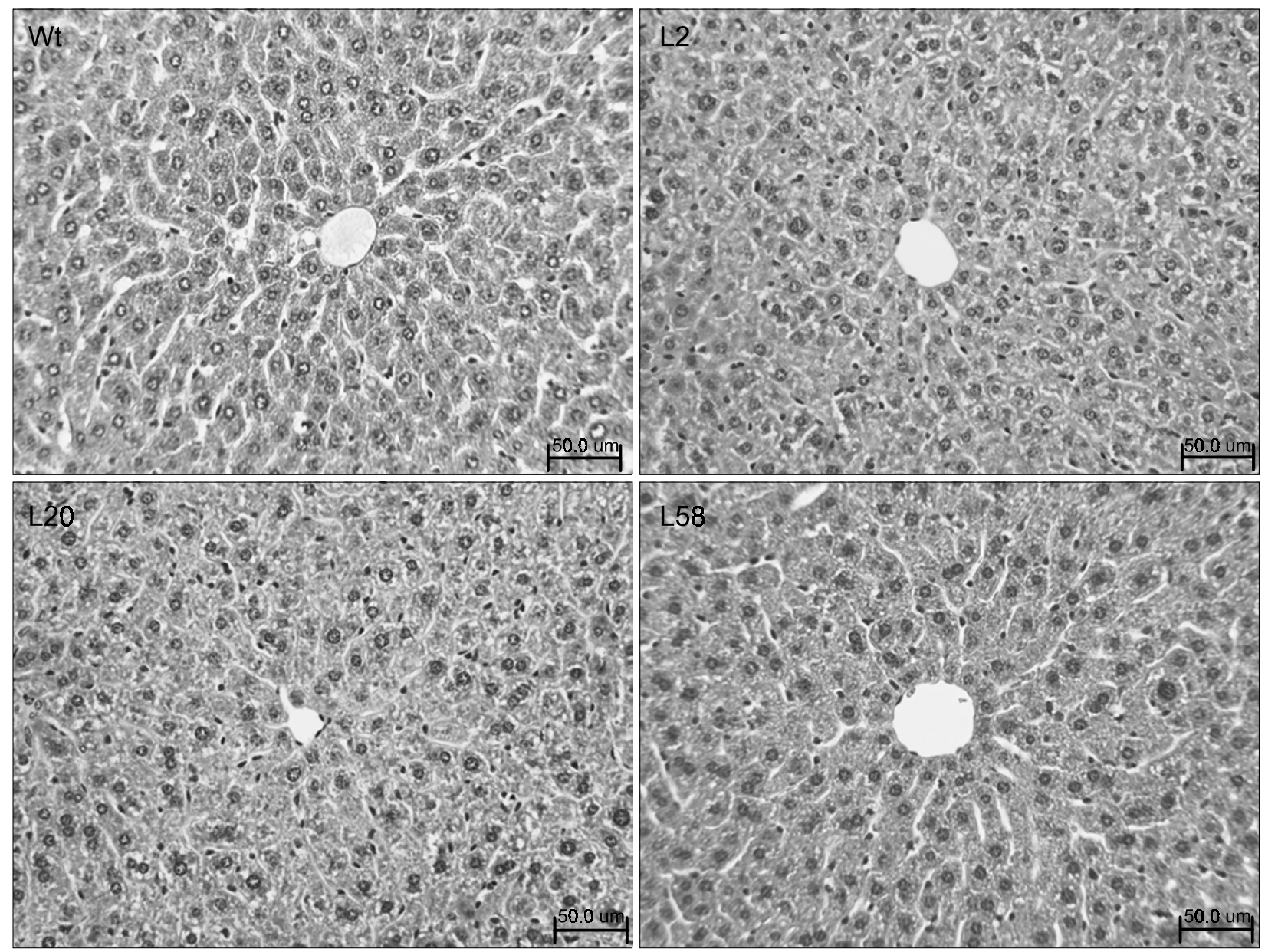

Figure 3. Histological analysis of livers from normal and transgenic mice. Hematoxylin and eosin-stained paraffin sections of liver histology from normal littermates (Wt) and from transgenic lineages 2 (L2), 20 (L20), and 58 (L58) are shown at $40 \times$.

\section{Histological examinations}

To detect whether NS4B was cytopathic to the transgenic mice, the mice were sampled between 3 and 18 months of age. As the NS4B transgene was found to be expressed in the transgenic mice tissues, the organ tissues including the liver were examined by histopathological analysis using H\&Estained sections in this study. A summary of the pathological assays is shown in Table 1. No pathological changes were detected in the spleen, kidney, heart, lung, brain, testis, or ovary. Inflammation and steatosis were rarely detected in the livers of transgenic mice or in normal control littermates during the experimental period. The histological assays of the three lineages are shown in Figure 3.

\section{Discussion}

The expression of transgenic NS4B at the mRNA 
and protein levels was confirmed in the three established transgenic lineages. However, the expression of NS4B was not cytopathic to liver tissue and to other cell types in these mice. Compared with the tumorigenesis and cell dysplasia in liver tissues of our previously reported HBX and S99Q transgenic mice, which were produced using the same HBV enhancer to induce the expression of transgenes (Yu et al., 1999; Wang et al., 2004), the non-pathologic changes detected in our NS4B transgenic lineages indicated a non-cytopathic function of NS4B in most cell types, even though the in vitro investigation showed the possibility of functions relating to the cell cycle (Florese et al., 2002) or transformation (Park et al., 2000, Qu et al., 2001).

An understanding of HCV-mediated pathogenesis and the control of HCV infection have been impaired in part because of the unavailability of an efficient cell culture system for virus growth and a convenient small animal model. Advances in the field of transgenic technology have offered us unique opportunities to develop valuable rodent models for human diseases (Majumder et al., 2003). Currently published data suggest that the expression of the $\mathrm{HCV}$ virus gene from the HCV genotype $1 \mathrm{~b}$ (Japanese strains) in the genetic background of the C57BL/6J mouse can induce hepatic alterations (Moriya et al., 1997; Moriya et al., 1998; Lerat et al., 2002). However, transgenic mice expressing the $\mathrm{HCV}$ gene that were established using another genotype of $\mathrm{HCV}$ or another genetic background of mouse strain (Kawamura et al., 1997; Pasquinelli et al., 1997; Majumder et al., 2003; Wang et al., 2004) failed to generate hepatic alterations. In Japan, approximately 15,90 , and $80 \%$ of patients with sporadic acute hepatitis, post-blood transfusion chronic hepatitis, and HCC, respectively, are positive for HCV infection (Watashi et al., 2003). The HCV genotype $1 \mathrm{~b}$ (Japanese strains) is the main causative agent for liver diseases. The collective data suggest that the genotype of the $\mathrm{HCV}$ and the mouse strain should be carefully selected when investigating the possible mechanisms related to $\mathrm{HCV}$ proteins. If the genetic background is proven to be an important factor in determining the disease expression in HCV-transgenic mice, it may provide useful clues to the mechanisms underlying the variable risk of disease in persons with chronic $\mathrm{HCV}$ infection (Lerat et al., 2002). In this study, even the genotype HCV 1b NS4B (Korean strains) did not show cytopathic effects on cells in vivo in the C57BL/6J genetic background, but other HCV genotypes of NS4B remain to be elucidated.

The HCV Core transgenic mice suggested that core protein may play an important role in HCVinduced steatosis and HCC (Moriya et al., 1997;
Moriya et al., 1998; Shin et al., 2005). Recently, Lerat et al. suggested that the expression of the structural proteins enhances a low background of steatosis in C57BL/6 mice, while the additional low-level expression of the nonstructural proteins increases the risk of cancer (Lerat et al., 2002). Among the nonstructural proteins, NS5A and NS4B are the primary candidates, but their functions are largely unknown. It has been reported that transgenic mice expressing NS5A protein did not show any pathological changes (Majumder et al., 2003), which indicates that NS5A protein alone is not cytopathic or oncogenic to hepatocytes. In this study, we first screened for possible pathological functions, which we inferred, of the NS4B protein in vivo. Mice in the three established transgenic lineages that expressed the NS4B transgene constitutively in transgenic tissues did not show any pathological changes. However, the occurrence of cooperative function with other virus proteins in relation to liver disease remains to be elucidated.

In conclusion, our investigation indicated that the HCV NS4B protein is not a causative agent of liver disease in our transgenic model. However, the possibility of cooperative functions with other viral proteins or causative agents remains to be elucidated.

\section{Acknowledgement}

This work was supported by the $21^{\text {st }}$ Century Frontier Program in the Functional Human Genome Project of Korea (grant no. HGC0300324).

\section{References}

Chisari FV. Hepatitis B virus transgenic mice: insights into the virus and the disease. Hepatology 1995;22:1316-25

Florese RH, Nagano-Fujii M, Iwanaga Y, Hidajat R, Hotta H. Inhibition of protein synthesis by the nonstructural proteins NS4A and NS4B of hepatitis C virus. Virus Res 2002;90: 119-31

Frith $\mathrm{CH}$, Ward JM. A morphologic classification of proliferative and neoplastic hepatic lesions in mice. J Environ Pathol Toxicol 1979; 3:329-51

Hanahan D. Dissecting multistep tumorigenesis in transgenic mice. Annu Rev Genet 1988;22:479-519

Hanley, KA, Manlucu LR, Gilmore LE, Blaney Jr JE, Hanson $C T$, Murphy BR, Whitehead SS. A trade-off in replication in mosquito versus mammalian systems conferred by a point mutation in the NS4B protein of dengue virus type 4 . Virology 2003;312:222-32

Kato J, Kato N, Yoshida H, Ono-Nita SK, Shiratori Y, Omata M. Hepatitis C virus NS4A and NS4B proteins suppress translation in vivo. J Med Virol 2002;66:187-99 
Kawamura T, Furusaka A, Koziel MJ, Chung RT, Wang TC, Schmidt EV, Liang TJ. Transgenic expression of hepatitis C virus structural proteins in the mouse. Hepatology 1997;25: 1014-21

Lemon SM, Chisari FV, Lai MM, Nishioka K, Mishiro S, Johnson L. The Nineteenth United States-Japan Joint Hepatitis Panel Meeting. Hepatology 1998;28:881-7

Lerat H, Honda M, Beard MR, Loesch K, Sun J, Yang Y, Okuda M, Gosert R, Xiao SY, Weinman SA, Lemon SM. Steatosis and liver cancer in transgenic mice expressing the structural and nonstructural proteins of hepatitis $C$ virus. Gastroenterology 2002;122:352-65

Lundin M, Monne M, Widell A, Von Heijne G, Persson MA. Topology of the membrane-associated hepatitis $C$ virus protein NS4B. J Virol 2003;77:5428-38

Majumder M, Steele R, Ghosh AK, Zhou XY, Thornburg L, Ray $R$, Phillips NJ, Ray RB. Expression of hepatitis $C$ virus nonstructural $5 \mathrm{~A}$ protein in the liver of transgenic mice. FEBS Lett 2003;555:528-32

Members of the European Association for the Study of the Liver, 1999. J Hepatol 1999;30 Suppl 1:311-31

Milich DR. Transgenic technology and the study of hepatitis viruses: a review of what we have learned. Can J Gastroenterol 2000;14:781-7

Moriwaki $\mathrm{H}$. Prevention of liver cancer: basic and clinical aspects. Exp Mol Med 2002;34:319-25

Moriya K, Fujie H, Shintani $Y$, Yotsuyanagi H, Tsutsumi T, Ishibashi K, Matsuura Y, Kimura S, Miyamura T, Koike K. The core protein of hepatitis $C$ virus induces hepatocellular carcinoma in transgenic mice. Nat Med 1998;4:1065-7

Moriya $\mathrm{K}$, Yotsuyanagi $\mathrm{H}$, Shintani $\mathrm{Y}$, Fujie $\mathrm{H}$, Ishibashi $\mathrm{K}$, Matsuura Y, Miyamura T, Koike K. Hepatitis $C$ virus core protein induces hepatic steatosis in transgenic mice. J Gen Virol 1997; 78 (Pt 7):1527-31

National Institutes of Health Consensus Development Conference Panel statement: management of hepatitis $C$. Hepatology 1997; 26:2S-10S

Park, JS, Yang JM, Min MK. Hepatitis C virus nonstructural protein NS4B transforms NIH3T3 cells in cooperation with the
Ha-ras oncogene. Biochem Biophys Res Commun 2000; 267:581-7

Pasquinelli C, Shoenberger JM, Chung J, Chang KM, Guidotti LG, Selby M, Berger K, Lesniewski R, Houghton M, Chisari FV. Hepatitis $\mathrm{C}$ virus core and $\mathrm{E} 2$ protein expression in transgenic mice. Hepatology 1997;25:719-27

Piccininni S, Varaklioti A, Nardelli M, Dave B, Raney KD, McCarthy JE. Modulation of the hepatitis $C$ virus RNAdependent RNA polymerase activity by the non-structural (NS) 3 helicase and the NS4B membrane protein. J Biol Chem 2002;277:45670-9

Qu L, McMullan LK, Rice CM. Isolation and characterization of noncytopathic pestivirus mutants reveals a role for nonstructural protein NS4B in viral cytopathogenicity. J Virol 2001;75:10651-62

Shin JY, Hur W, Wang JS, Jang JW, Kim CW, Bae SH, Jang SK, Yang SH, Sung YC, Kwon OJ, Yoon SK. HCV core protein promotes liver fibrogenesis via up-regulation of CTGF with TGF-ß1. Exp Mol Med 2005; 37:138-45

Tan SL, Katze MG. How hepatitis C virus counteracts the interferon response: the jury is still out on NS5A. Virology 2001; 284:1-12

Wang AG, Moon HB, Lee YH, Yu SL, Kwon HJ, Han YH, Fang W, Lee TH, Jang KL, Kim SK, Yu DY, Lee DS. Transgenic expression of Korean type hepatitis $\mathrm{C}$ virus core protein and related mutants in mice. Exp Mol Med 2004; 36:588-93

Watashi K, Shimotohno K. The roles of hepatitis C virus proteins in modulation of cellular functions: a novel action mechanism of the HCV core protein on gene regulation by nuclear hormone receptors. Cancer Sci 2003;94:937-43

Westaway, EG, Khromykh AA, Kenney MT, Mackenzie JM, and Jones MK. Proteins $C$ and NS4B of the flavivirus Kunjin translocate independently into the nucleus. Virology 1997; 234:31-41

Yu, DY, Moon HB, Son JK, Jeong S, Yu SL, Yoon H, Han YM, Lee CS, Park JS, Lee CH, Hyun BH, Murakami S, Lee KK. Incidence of hepatocellular carcinoma in transgenic mice expressing the hepatitis B virus X-protein. J Hepatol 1999. 31:123-32 